\title{
The LMC H II region N 214C and its peculiar nebular blob ${ }^{\star \star \star}$
}

\author{
F. Meynadier ${ }^{1}$, M. Heydari-Malayeri ${ }^{1}$, and N. R. Walborn ${ }^{2}$ \\ 1 LERMA, Observatoire de Paris, 61 avenue de l'Observatoire, 75014 Paris, France \\ e-mail: Frederic.Meynadier@obspm.fr \\ 2 Space Telescope Science Institute, 3700 San Martin Drive, Baltimore, Maryland 21218, USA
}

Received 15 December 2004/ Accepted 3 March 2005

\begin{abstract}
We study the Large Magellanic Cloud H II region N214C using imaging and spectroscopy obtained at the ESO New Technology Telescope. On the basis of the highest resolution images so far obtained of the OB association LH 110, we show that the main exciting source of the $\mathrm{H}$ II region, $\mathrm{Sk}-71^{\circ} 51$, is in fact a tight cluster of massive stars consisting of at least 6 components in an area $\sim 4^{\prime \prime}$ wide. Spectroscopic observations allow us to revise the spectral type of the main component (\# 17) to $\mathrm{O} 2 \mathrm{~V}\left(\left(\mathrm{f}^{*}\right)\right)+\mathrm{OB}$, a very rare, hot type. We also classify several other stars associated with $\mathrm{N} 214 \mathrm{C}$ and study the extinction and excitation characteristics of the $\mathrm{H}$ II region. Moreover, we obtain $B V R$ photometry and astrometry of 2365 stars and from the corresponding color-magnitude diagram study the stellar content of N 214C and the surrounding LH 110. Furthermore, we discover a striking compact blob of ionized gas in the outer northern part of $\mathrm{N} 214 \mathrm{C}$. A spherical structure of $\sim 5^{\prime \prime}$ in radius $(\sim 1.3 \mathrm{pc})$, it is split into two lobes by absorbing dust running diametrically through its center. We discuss the possible nature of this object.
\end{abstract}

Key words. stars: early-type - ISM: individual objects: N 214C - galaxies: Magellanic Clouds

\section{Introduction}

Among the Large Magellanic Cloud (LMC) H II regions catalogued by Henize (1956), N 214 is one of the southernmost, lying below the bar, at a distance of $\sim 135^{\prime}(\sim 2.1 \mathrm{kpc}$ in projection) from the famous 30 Doradus (assuming a distance modulus of $18.6 \mathrm{mag}$, Groenewegen \& Oudmaijer 2000). N 214 appears as an elongated structure, $\sim 15^{\prime} \times 4^{\prime}(\sim 220 \mathrm{pc} \times 60 \mathrm{pc})$, composed of at least 8 nebular components (A to $\mathrm{H}$ ), most of them very dim. This gas complex is a noteworthy region of ongoing star formation, as suggested by the detection of molecular emission toward components N214C and N 214DE (Israel et al. 1993; Heikkila et al. 1998). The latter molecular cloud is of particular interest since it is larger, more intense, and moreover contains several molecular species $\left(\mathrm{HCN}, \mathrm{HCO}^{+}\right.$, CS, etc.) tracing high-density regions (Chin et al. 1997). In fact the H I column density toward N214 is one of the largest in the whole LMC (Israel 1997). N 214 also harbors the OB association LH 110 (Lucke \& Hodge 1970), a study of which was presented by Oey (1996).

Few works have been devoted to the H II regions constituting N 214 and its associated stellar populations. The present

^ Based on observations obtained at the European Southern Observatory, La Silla, Chile; Program 69.C-0286(A) and 69.C-0286(B).

$\star \star$ Tables 2 and 3 are only available in electronic form at the CDS via anonymous ftp to cdsarc.u-strasbg. fr (130.79.128.5) or via http://cdsweb.u-strasbg.fr/cgi-bin/qcat?J/A+A/436/117 paper deals with the brightest component N 214C, also called NGC 2103 or DEM L 293 (Davies et al. 1976). We use optical imaging and spectroscopy in order to study the H II region as well as its associated stars. $\mathrm{N} 214 \mathrm{C}$ is quite attractive since it hosts $\mathrm{Sk}-71^{\circ} 51$, a very hot and massive star previously classified O3 V((f*)) (Walborn et al. 2002), which our present work shows to be even earlier. The most massive stars being particularly rare, and since we do not know yet how they form, N 214C provides an excellent opportunity for studying the formation site of such a star, including its related stellar populations.

\section{Observations and data reduction}

\subsection{Sub-arcsecond imaging and photometry}

N214C was observed on 28 September 2002 using the ESO New Technology Telescope (NTT) equipped with the active optics and the SUperb Seeing Imager (SuSI2). The detector was made up of two CCD chips, identified as ESO \#45 and \#46. The two resulting frames were automatically combined in a single FITS file, whereas the space between the two chips was "filled" with some overscan columns so that the respective geometry of the two chips was approximatively preserved. The gap between the chips corresponds to $\sim 100$ real CCD pixels, or $\sim 8$ ". The file format was $4288 \times 4096$ pixels, and the measured pixel size 0 ' 085 on the sky. Each chip of the mosaic covered a field of 5.5 $\times 2$ '.7. See the ESO manual SuSI2 for more technical information (LSO-MAN-ESO-40100-0002/1.9). 
Nebular imaging was carried out using the narrow band filters centered on the emission lines $\mathrm{H} \alpha$ (ESO \#884), $\mathrm{H} \beta$ (\#881), and [O III] (\#882) with basic exposures of $300 \mathrm{~s}$; for the first two filters 2 exposures and in the latter case 4 exposures were obtained. The image quality was quite good during the night, as represented by a seeing of $0 .{ }^{\prime} 5-0 . ' 8$.

Photometry was performed in the $B V R$ Bessell system using the filters ESO \#811 $(B), \# 812(V)$, and \#813 $(R)$. We were particularly careful to keep most of the brightest stars in the field under the detector's saturation level in order to get high quality Point Spread Functions (PSF). This led us to adopt unit exposure times of $60 \mathrm{~s}$ for $B$ and $V$ and $30 \mathrm{~s}$ for $R$ respectively. The exposures for each filter were repeated 4 times using ditherings of $5^{\prime \prime}-10^{\prime \prime}$ for bad pixel rejection.

Seven standard stars, belonging to two Landolt photometric groups (SA 92 and T Phe) were observed at four different airmasses. This led to the determination of the photometry coefficients and zero-points using the photcal package under iraf. Those coefficients are in good agreement with the indicative values which are displayed on the SuSI2 web page.

The offset between standard stars' wide-aperture photometry and field stars' PSF-fitting photometry was calculated as follows: starting from one of the flat-fielded frames, we subtracted all stars except the ones used for determining the PSF with the daophot. substar procedure, using our preliminary DAOPHOT photometry and the corresponding PSF. This leads to a frame with only a few bright, isolated stars plus residues from the subtraction. We then performed both aperture and PSF-fitting photometry on those stars, using the same aperture we used for standard stars. Finally, we compared the results and eliminated deviant measurements (occasional bad PSF subtraction and strongly variable nebular background). This results in aperture corrections of $0.02,0.04$ and $0.03 \mathrm{mag}$ in $B, V$ and $R$ respectively.

During the photometry process, some slight discrepancies between the intensity of the frames were found: this effect was considered to be the consequence of episodic variations of sky transparency by $7 \%$ at most. In order not to introduce a systematic underestimation of the star magnitudes when averaging the frames, we decided to perform photometry on each individual frame with daophot. Then, for each star, we computed the magnitude differences from one frame to another, and deduced the mean magnitude shift between each frame. Choosing the brightest ones as the reference for each filter, we multiplied the three others by the appropriate correction factor $\left(10^{-0.4 \cdot \Delta m}\right.$, with $\Delta m$ being the (negative) mean difference of magnitude between the brightest frame and the current frame) and performed another run of photometry. By cross-correlating the positions of the sources in the various photometry files, we obtained the mean magnitude (average of the $4 \mathrm{mag}$ ) and a decent estimator of the uncertainty on this magnitude (difference between maximum and minimum magnitudes, the sample being too small for $\sigma$ to be significant). The mean uncertainties, much larger and probably more meaningful than the daophot internal errors, are reported in Table 1 . Finally, the process yielded the photometry of 2321 stars in all three filters (online Table 2).

A composite, six-color image of the whole N 214C field is displayed in Fig. 1, while Fig. 2 shows a subfield in $V$, where
Table 1. Mean overall photometric errors.

\begin{tabular}{cccc}
\hline \hline Mag & $B$ & $V$ & $R$ \\
\hline 14 & 0.007 & 0.014 & 0.018 \\
15 & 0.012 & 0.016 & 0.021 \\
16 & 0.014 & 0.016 & 0.022 \\
17 & 0.019 & 0.025 & 0.030 \\
18 & 0.029 & 0.031 & 0.040 \\
19 & 0.044 & 0.047 & 0.066 \\
20 & 0.078 & 0.082 & 0.107 \\
21 & 0.149 & 0.144 & 0.222 \\
\hline
\end{tabular}

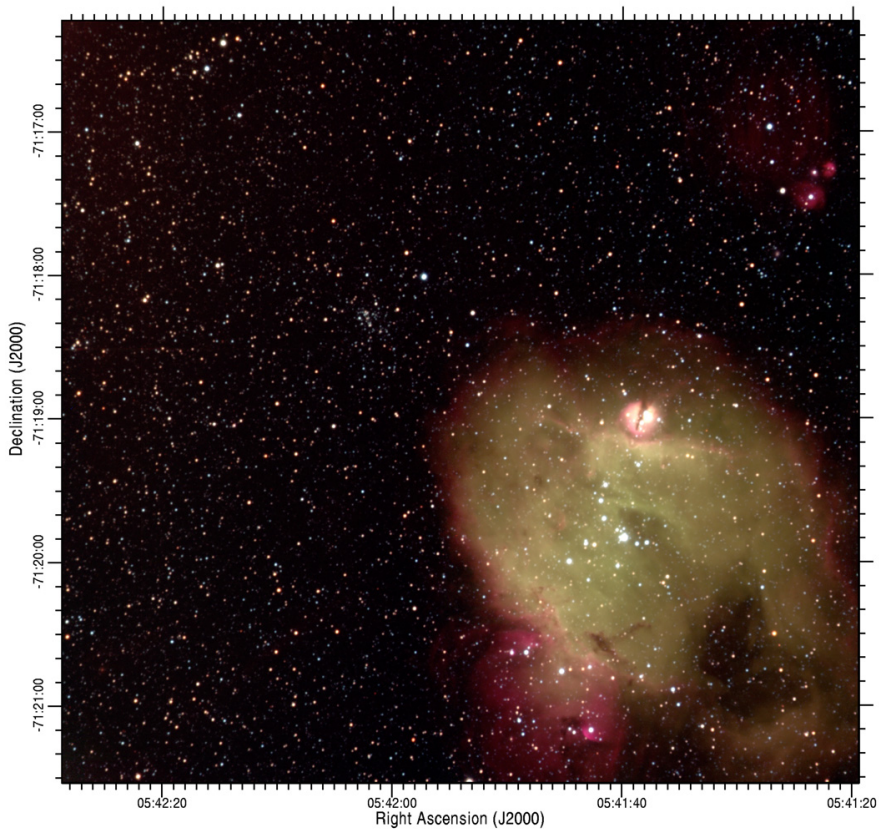

Fig. 1. A composite six-color image of the LMC H II region N 214C and the OB association LH 110 in which it lies. The image, taken with the ESO NTT/SuSI2, results from the co-addition of narrow and broad-band filters $R$ and $\mathrm{H} \alpha$ (red), $V$ and [O III] (green), and $B$ and $\mathrm{H} \beta$ (blue). The field size is $364^{\prime \prime} \times 348^{\prime \prime}$ corresponding to $91 \times 87 \mathrm{pc}$. North is up and east to the left. The brightest star, situated toward the middle of the nebula, is the $\mathrm{Sk}-71^{\circ} 51$ cluster. The striking compact H II blob lies $\sim 60^{\prime \prime}$ ( $\sim 15 \mathrm{pc}$ ) north of Sk $-71^{\circ} 51$. See Fig. 7 for identifications of the compact nebulae lying north-west of N 214C.

the most conspicuous stars are labelled. The corresponding color-magnitude diagram is displayed in Fig. 3, and discussed in Sect. 5.

\subsection{Image restoration}

Sk $-71^{\circ} 51$, the most prominent star of the field, lies in a crowded area. In order to obtain reliable photometry for this star, we performed a deconvolution on a subfield centered on the star. We used the MCS deconvolution algorithm, originally proposed by Magain et al. (1998). The strength of this code is that it takes the finite resolution of CCDs into account and ensures that the Shannon sampling theorem is not violated during the deconvolution process: in particular the final PSF is chosen so that it remains well sampled by the pixels $(F W H M \geq$ 2 pixels). This allows accurate photometry and astrometry with 


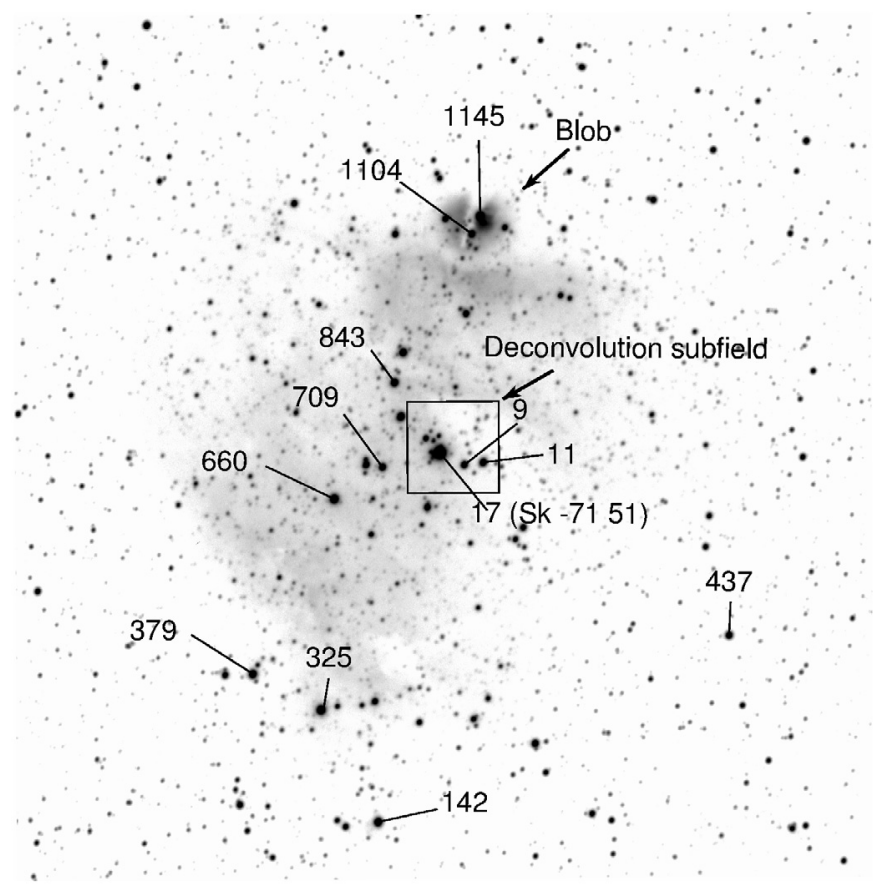

Fig. 2. The H II region $\mathrm{N} 214 \mathrm{C}$ through the broad-band filter $V$ obtained using ESO NTT equipped with SuSI2. North is up and east to the left. Field size: $206^{\prime \prime} \times 208^{\prime \prime}(\sim 52 \times 52 \mathrm{pc})$. The stars for which spectroscopy has been obtained are labelled, as well as some noteworthy stars of the sample indicated in Fig. 3. The subfield $\left(21^{\prime \prime} 8^{2}\right)$ on which the deconvolution has been carried out is also shown.

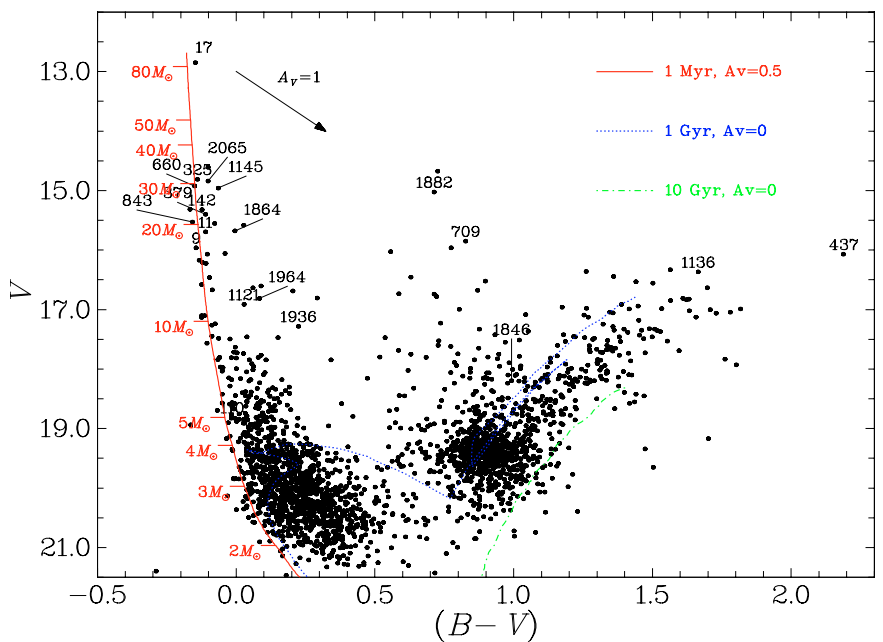

Fig. 3. Color-magnitude, $V$ versus $B-V$, diagram for the 2341 stars observed toward LMC N 214C. Three isochrones are shown, $1 \mathrm{Myr}$ with $A_{\mathrm{V}}=0.5 \mathrm{mag}$ (red curve), $1 \mathrm{Gyr}$ (dotted blue), and $10 \mathrm{Gyr}$ (dashed-dotted green), computed for a metallicity of $Z=0.008$ (Lejeune \& Schaerer 2001) and a distance modulus of $18.6 \mathrm{mag}$. A number of masses between 2 and $80 M_{\odot}$ are indicated for the $1 \mathrm{Myr}$ isochrone. The arrow indicates the reddening shift for $A_{\mathrm{V}}=1 \mathrm{mag}$. The numbers refer to the stellar identifications presented in Fig. 2.

minimum deconvolution artefacts for any blended sources, provided that the PSF of the original image is adequately oversampled and that the $S / N$ ratio of the sources to be deconvolved remains high enough. We had successfully used this algorithm, in its original implementation, for one of our previous works
(Heydari-Malayeri et al. 2003). The present work makes use of an improved version of the MCS algorithm, allowing simultaneous PSF determination and photometry in dense stellar fields (Magain et al. 2005).

The original data are a set of 12 frames, $256^{2}$ pixels each $\left(21^{\prime \prime} 8^{2}\right.$ on the sky) extracted from the $B, V$ and $R$ individual flat-fielded frames described earlier. Unfortunately 2 of the $4 V$ frames were found to be problematic: one because of a meteoric trace close to faint sources, and the other because of saturation of a few pixels. We conservatively chose not to consider those two frames for deconvolution, which reduces the set to: $4 \mathrm{~B}$ frames, $2 \mathrm{~V}$ frames, and $4 R$ frames. After subtraction of the nebular background using sextractor (Bertin \& Arnouts 1996), the 10 frames have been processed simultaneously: the astrometry was assumed to be identical on each frame, with a small relative shift. Finally the output consists of 10 photometry values and a pair of coordinates for each source: the mean value in each filter is computed afterwards, the spread of values giving an estimate of the uncertainty on instrumental magnitudes with this method. As a result, the subfield is resolved into 48 stars, labelled from 1 to 48 (Fig. 4), the photometry of 44 among them is given in online Table 3.

\subsection{Spectroscopy}

The EMMI spectrograph attached to the ESO NTT telescope was used on 28 and 30 September 2002 to obtain several long slit spectra of the stars. The grating was \# 12 centered on $4350 \AA$ (BLMRD mode) and the detector was a Tektronix CCD TK1034 with $1024^{2}$ pixels of size $24 \mu \mathrm{m}$. The covered wavelength range was $3810-4740 \AA$ and the dispersion $38 \AA \mathrm{mm}^{-1}$, giving $F W H M$ resolutions of $2.70 \pm 0.10$ pixels or $2.48 \pm 0.13 \AA$ for a $1^{\prime \prime}$.0 slit. At each position we first took a short $5 \mathrm{~min}$ exposure followed by one or two longer $15 \mathrm{~min}$ exposures. The instrument response was derived from observations of the calibration stars LTT 7379, LTT 6248, and LTT 7987.

The seeing conditions were very good, varying between 0.4 and 0.5 . The identification of the stars along the slits was based on monitor sketches drawn during the observations. However, in order to avoid ambiguous identifications more especially in crowded regions, we also worked out a small IRAF task, using the position angle information in the FITS headers. First, each spectrum was integrated along the dispersion axis, the result being stored into a two-pixel wide strip, which is close to the actual size of the slit. Then, the position angle and the pixel-arcsec correspondence were used to calculate the rotation matrix for the World Coordinate System (WCS). This allowed creation of a slit chart, an $\alpha-\delta$ calibrated two-dimensional image containing accurate slit orientations. Displaying simultaneously the slit chart beside the SuSI images and using the WCS correlations it was possible to accurately check the identity of the star in the slit.

EMMI was also used to obtain nebular spectra with grating \# 8 (4550-6650 $\AA$ ) in the REMD mode. The detector was CCD \# 63, MIT/LL, $2048 \times 4096$ pixels of $15^{2} \mu \mathrm{m}^{2}$ each. A number of spectra were obtained with the slit set in east-west 

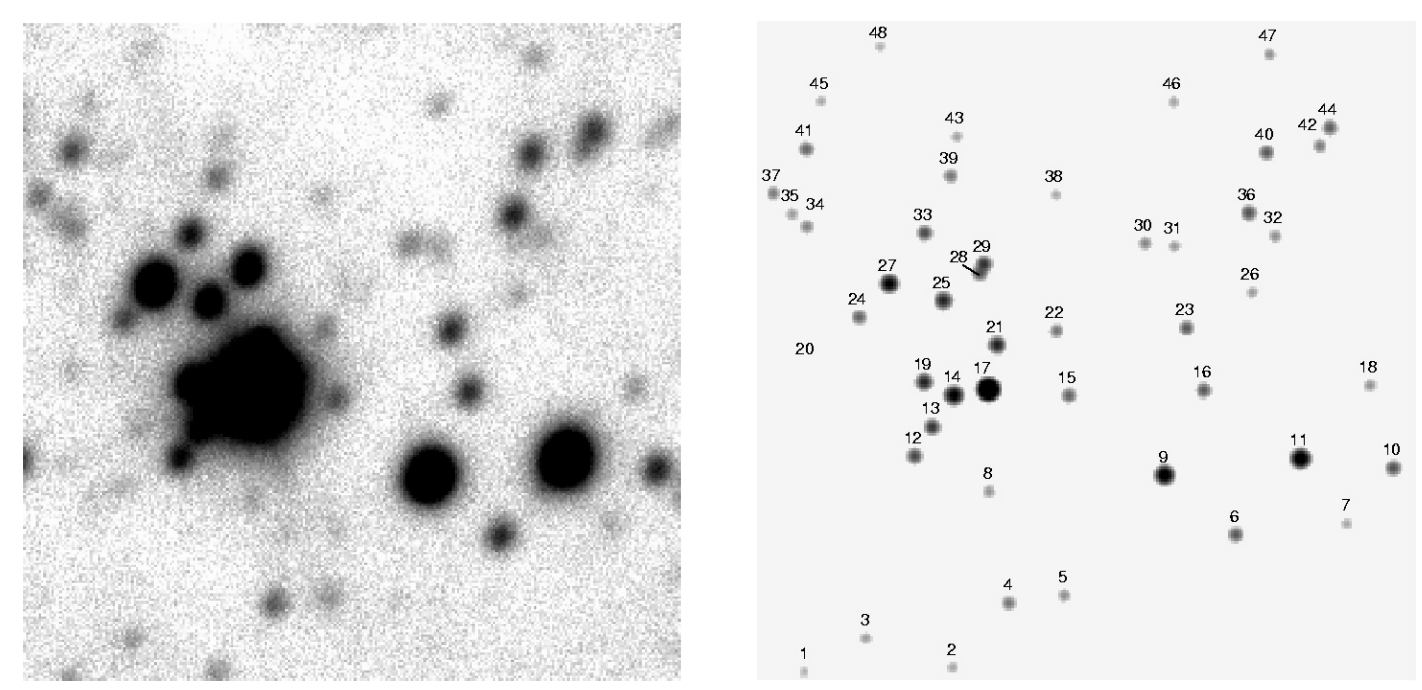

Fig. 4. The deconvolution subfield around the hot star $\mathrm{Sk}-71^{\circ} 51$ in $V$ (see Fig. 2). Left: single frame after subtraction of the nebular background, exposure time $60 \mathrm{~s}$, seeing about 8.5 pixels ( 0 '. $^{\prime}$ ). Right: the same field after deconvolution. The resulting $F W H M$ of the sources is 3 pixels, or 0 ' 25 on the sky. Field size $21^{\prime \prime} \cdot 7^{2}\left(\sim 5.4^{2} \mathrm{pc}\right)$. North is up and east to the left.

and north-south orientations using a basic exposure time of $300 \mathrm{~s}$ repeated several times. These spectra were used to calibrate the line ratio maps $\mathrm{H} \alpha / \mathrm{H} \beta$ and $[\mathrm{O} \mathrm{III}] / \mathrm{H} \beta$ which are based on imaging (see Sects. 3.2 and 3.3).

The stellar spectra were extracted using the specres iraf task (Lucy \& Walsh 2003), part of the stecf iraf package (http://www.stecf.org/software/stecf-iraf/). This procedure uses an iterative technique involving LucyRichardson restoration to avoid contamination from the inhomogeneous nebular background.

\section{Results}

\subsection{General morphology}

$\mathrm{N} 214 \mathrm{C}$ is the most prominent $\mathrm{H} \alpha$ emission nebula of the $\mathrm{OB}$ association LH 110 (Fig. 1). It extends over $\sim 3.5$ ( 52 pc) along the north-south direction while its mean size along eastwest is less than 2'.5 (38 pc), becoming narrower in its southern part. The bulk of stars making up LH 110 is visible in Fig. 1, and in particular a tight cluster can be seen around $\alpha=05: 42: 02, \delta=-71: 18: 20$.

At the center of the nebula lies $\mathrm{Sk}-71^{\circ} 51$, the region's brightest and hottest star (Walborn et al. 2002). At a distance of $\sim 15^{\prime \prime}$ north of $\mathrm{Sk}-71^{\circ} 51$ runs a long arc of shocked gas with a curvature pointing to the star. There are a dozen less bright stars scattered across the nebula and mainly around $\mathrm{Sk}-71^{\circ} 51$. The green color in the composite image (Fig. 1) covering the bulk of the $\mathrm{HII}$ region comes from the high excitation forbidden line [O III] $\lambda 5007$. Moreover, several fine, filamentary structures and fine absorption pillars and features are visible. The eastern or more particularly the south-eastern boundaries of the $\mathrm{H}$ II region exhibit a rim structure, evoking a cavity filled with ionized gas. There is however a nebular extension toward the south-east where three relatively bright stars are present (\# 325, \# 379, \# 142) and the emission is mostly due to $\mathrm{H} \alpha$. We also see a relatively large loop of ionized gas which protrudes from the

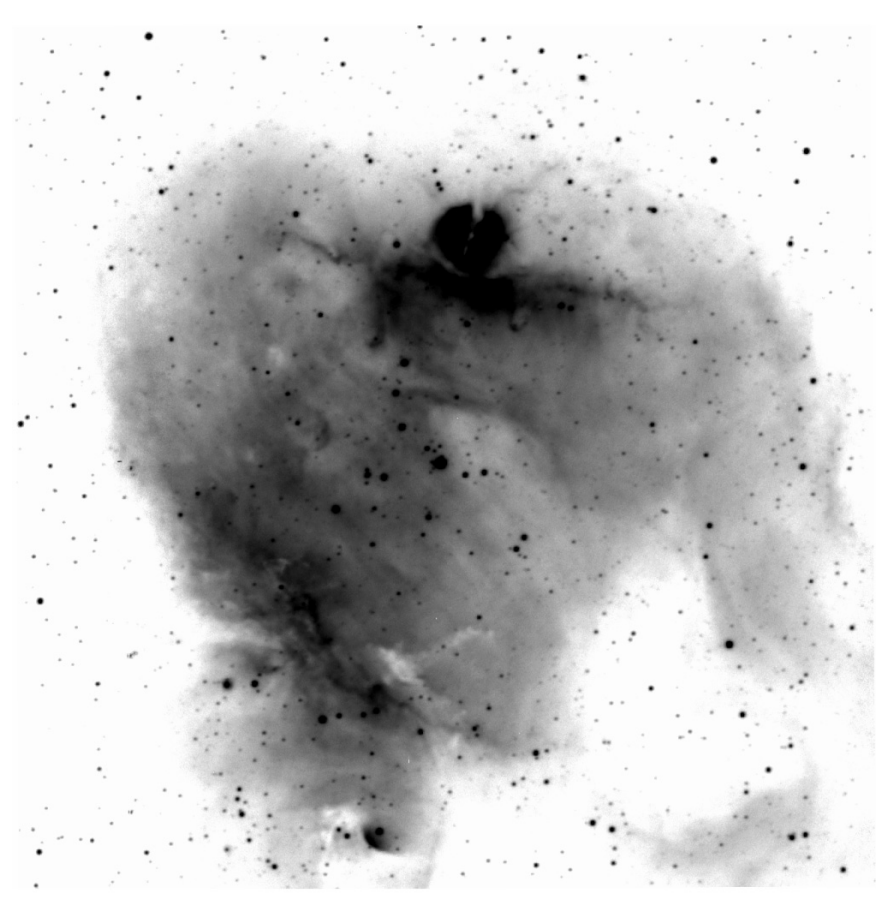

Fig. 5. An $\mathrm{H} \alpha$ image with a deep intensity cut displaying the northern and southern-eastern ridges. Field size the same as in Fig. 2.

north-west and bends to join the main body of the nebula at its southern part.

An $\mathrm{H} \alpha$ image with much deeper cut Fig. 5 shows a remarkable symmetry of the nebular structures with respect to $\mathrm{Sk}-71^{\circ} 51$ as far as the bright ridges to the north and south are concerned. The H II region appears to be "breaking out", i.e. encountering lower densities, to the east and west. There may be dense clouds north and south.

A remarkable feature of $\mathrm{N} 214 \mathrm{C}$ is the presence of a globular blob of ionized gas at $\sim 60^{\prime \prime}(\sim 15 \mathrm{pc}$ in projection) north of $\mathrm{Sk}-71^{\circ} 51$ to which attention was drawn by Walborn et al. (2002). It appears as a sphere of radius $\sim 5^{\prime \prime}(\sim 1.3 \mathrm{pc})$ split into 


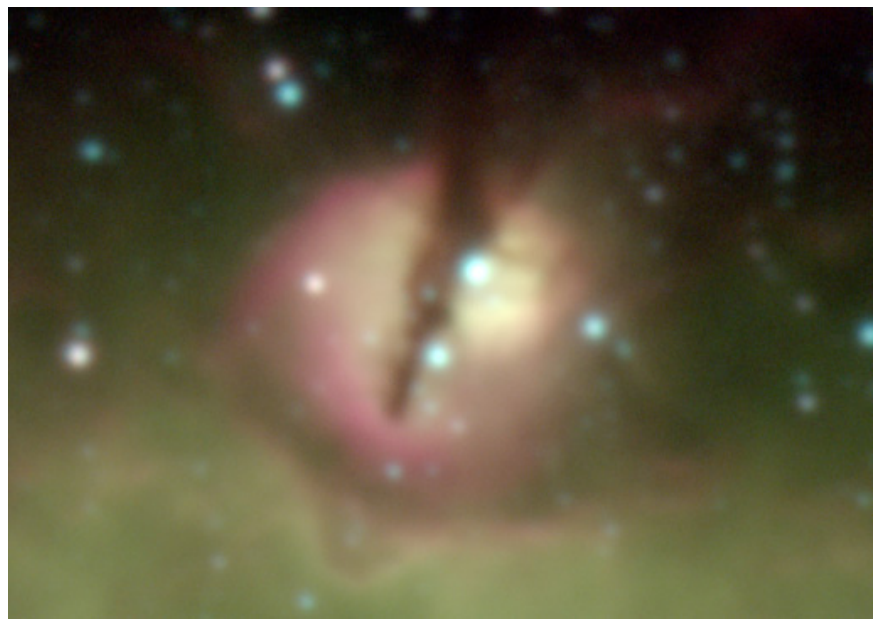

Fig. 6. A composite color image of the nebular blob lying $\sim 60^{\prime \prime}$ $(15 \mathrm{pc})$ north of the $\mathrm{Sk}-71^{\circ} 51$ cluster. Based on individual images taken through narrow-band filters $\mathrm{H} \alpha$ (red), [O III] (green) and $\mathrm{H} \beta$ (blue). Size $513 \times 363$ pixels, $43^{\prime \prime} \times 31^{\prime \prime}$ on the sky, corresponding to $\sim 11 \times 8 \mathrm{pc}$. North is up and east to the left. The brightest star north of the emission peak is \# 1145 and the one south of the peak is \# 1104 . The faint star situated toward the center of the blob is \# 1132. The two relatively bright stars in the eastern and western lobes are \# 1136 and \# 1121 respectively (see also Table 4).

two lobes by a dust lane which runs along an almost northsouth direction (Fig. 6). The absorption lane is $\sim 1^{\prime \prime}$ wide in its southern part and becomes larger toward the north, reaching a width of $\sim 3^{\prime \prime} .5(\sim 0.9 \mathrm{pc})$. The western lobe has a bright emission peak not only in the Balmer $\mathrm{H} \alpha$ and $\mathrm{H} \beta$ recombination lines, but also in the higher excitation [O III $] \lambda 5007$ line. Several stars are seen toward the face of the HII blob, the brightest one (\# 1145) appears to lie $\sim 2$ '. 4 ( $~ 0.6$ pc) north of the peak zone at the border of the dark lane. We see another conspicuous star (\# 1104) at $\sim 3 . \prime 9$ ( 1 pc) south of the emission peak. And there is also a faint star (\# 1132) lying apparently toward the center of the blob. More generally, the blob seems to be placed on a ridge of ionized gas. The ridge follows the structure of the blob implying a possible interaction, and runs on both sides of the blob over $\sim 80^{\prime \prime}$ on the sky, corresponding to $\sim 20 \mathrm{pc}$ in projection.

The observations also resolve the LHA $120-\mathrm{N} 214 \mathrm{H}$ component which lies north-west of N $214 \mathrm{C}$ at a distance of $\sim 130^{\prime \prime}$ $(\sim 33$ pc) from the blob (Fig. 7). We show that N214H consists in fact of four unknown, compact H II regions hosting exciting stars and a diffuse nebula surrounding star \# 2065. Tentatively, we call these anonymous nebulae N 214H-1, 2, 3, and 4 . N $214 \mathrm{H}-1$ has a structure identical to that of the blob, in miniature!

\subsection{Extinction}

Figure 8 displays the extinction map of the N 214C region obtained from the Balmer decrement $\mathrm{H} \alpha / \mathrm{H} \beta$ ratio. The most extincted part of the nebula is the absorption lane where the ratio reaches a value of $\sim 10$ corresponding to $A_{\mathrm{V}} \sim 3.5 \mathrm{mag}$. It should be underlined that this estimate is likely a lower limit

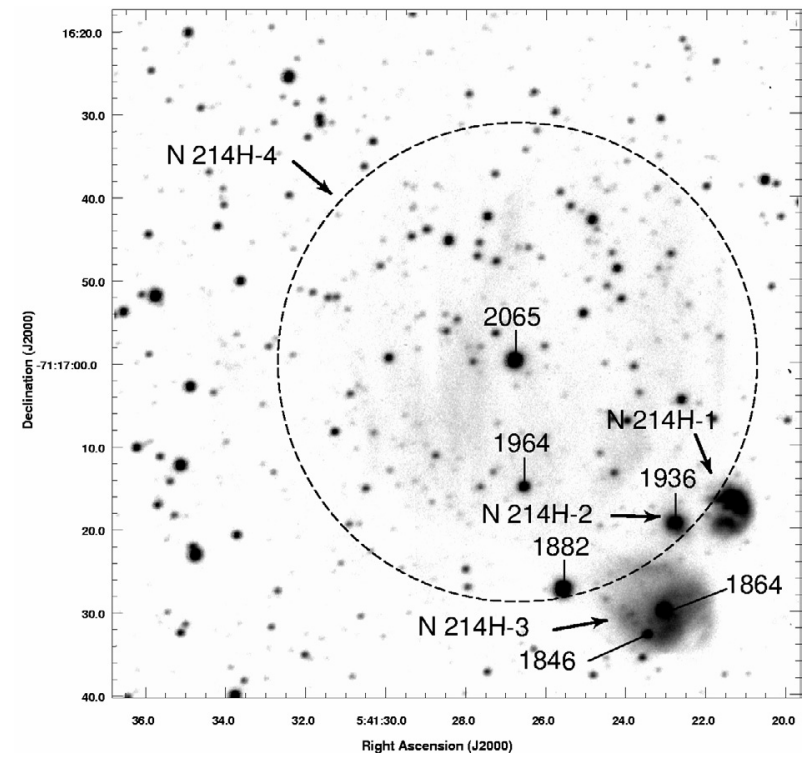

Fig. 7. $\mathrm{H} \alpha$ image of the anonymous compact nebulae, labelled $\mathrm{N} 214 \mathrm{H}-1,2$, and 3 and the diffuse $\mathrm{N} 214 \mathrm{H}-4$, all lying north-west of $\mathrm{N} 214 \mathrm{C}$. Field size $\sim 88^{\prime \prime} \times 88^{\prime \prime}(\sim 22 \times 22 \mathrm{pc})$. North is up and east to the left. See Table 4 for more information on the labelled stars.

because the $\mathrm{H} \alpha / \mathrm{H} \beta$ ratio samples outer zones. Moreover, the Balmer ratio is not a good extinction indicator in this particular case since the exciting star lies above the lane between the blob and the observer. Outside the lane, the ratio is on average $\sim 5.5\left(A_{\mathrm{V}}=1.8 \mathrm{mag}\right)$ and even reaches 6.2 $\left(A_{\mathrm{V}}=2.2 \mathrm{mag}\right)$ toward the bright peak of the western lobe. The ridge on which the blob apparently lies also has a relatively high ratio reaching sometimes $\sim 4.7\left(A_{\mathrm{V}}=1.4 \mathrm{mag}\right)$. Another notable reddened area is the south-eastern border of $\mathrm{N} 214 \mathrm{C}$ where the ratio fluctuates around $\sim 4\left(A_{\mathrm{V}}=0.9 \mathrm{mag}\right)$ and reaches as high as $\sim 4.3\left(A_{\mathrm{V}}=1.1 \mathrm{mag}\right)$. The ratio is significantly smaller and almost uniform toward other areas of the $\mathrm{H}$ II region and attains a value of $\sim 3\left(A_{\mathrm{V}}=0.1 \mathrm{mag}\right)$ in the area surrounding the hot star $\mathrm{Sk}-71^{\circ} 51$. The external loop structure also has a remarkable reddening, in average 3.5, corresponding to $A_{\mathrm{V}}=0.6 \mathrm{mag}$. Note that previous extinction estimates toward $\mathrm{N} 214 \mathrm{C}$ obtained through $4^{\prime} .9$ apertures yield the following global, smoothed results: $A(\mathrm{H} \alpha)=0.15 \mathrm{mag}, A(\mathrm{H} \alpha-$ radio $)=0.34 \mathrm{mag}, A(1500 \AA$-FIR $)=1.10 \mathrm{mag}$, and $A(1900 \AA$ FIR $)=0.66$ mag (Caplan \& Deharveng 1985, 1986; Bell et al. 2002).

\subsection{Excitation}

The [O III] $(5007 \AA) / \mathrm{H} \beta$ ratio (Fig. 8) has a uniform value of about 5 over an extended area centered on $\mathrm{Sk}-71^{\circ} 51$. In particular, the narrow nebular arc situated north of $\mathrm{Sk}-71^{\circ} 51$ can be distinguished by its slightly higher value of $\sim 5.5$. The ridge on which the blob seems to lie has a ratio between 4 and 5 .

Regarding the ionized blob, the western lobe has a higher $[\mathrm{O} \mathrm{III}] / \mathrm{H} \beta$ ratio attaining a value of 5.3 at most toward the bright emission area. This higher excitation zone is centered on star \# 1145 but cut by the dust lane. The eastern lobe has a 


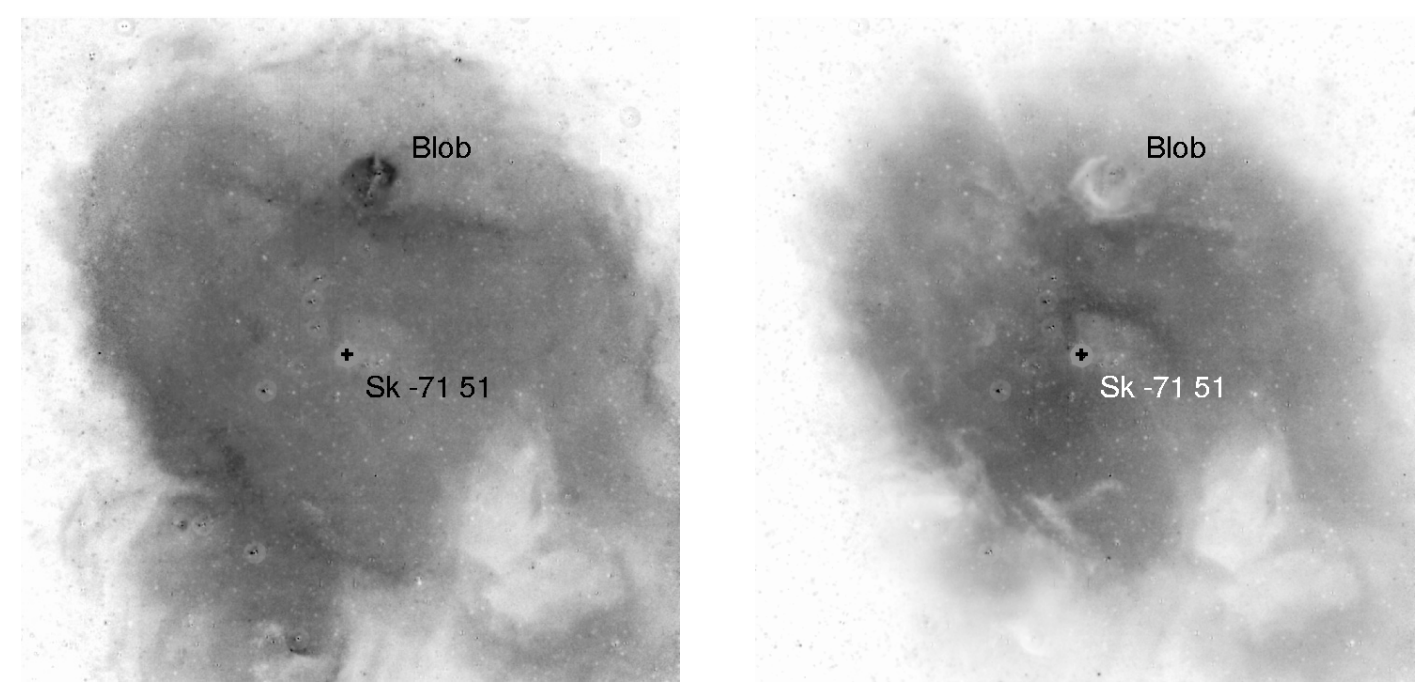

Fig. 8. Line ratio maps of the H II region N $214 \mathrm{C}$ based on narrow-band filter images, after subtraction of point sources with DAOPHOT/allstar. The white circles around the stars are processing artefacts. Left: the extinction $\mathrm{H} \alpha / \mathrm{H} \beta$ map. Right: the excitation $[\mathrm{O}$ III] $/ \mathrm{H} \beta$ map. Field size as in Fig. 2. North is up and east to the left.

smaller mean value of $\sim 3.5$ and rises to $\sim 3.8$ toward the northeastern part of the lobe.

\subsection{Multiplicity of $S k-71^{\circ} 51$}

Although it was known that $\mathrm{Sk}-71^{\circ} 51$ is not a single star but a compact cluster (Garmany \& Walborn 1987), its attributed luminosity was based on low-resolution observations obtained using a $61 \mathrm{~cm}$ telescope with an aperture $18^{\prime \prime}$ in size (Isserstedt 1975). Apart from the fact that $\mathrm{Sk}-71^{\circ} 51$ is a tight cluster, the presence of a relatively bright star (\#9), detached from the main cluster but possibly present in the aperture, leads to an overstimation of the magnitude.

The result of the image restoration by deconvolution, as explained in Sect. 2.2, for a $256^{2}$ pixels field, corresponding to $21^{\prime \prime} .8^{2}$ on the sky, centered on $\mathrm{Sk}-71^{\circ} 51$, is presented in Fig. 4 and listed in Table 3 which also gives the astrometry and photometry of the stars. The tight core of the $\mathrm{Sk}-71^{\circ} 51$ cluster, covering a $\sim 4$ " area, is made up of at least 6 components, stars \# 17, 14, 21, 19, 13, and 12. The brightest component, \# 17 with $V=12.85, B-V=-0.15$, and $V-R=-0.06 \mathrm{mag}$, is separated by $\sim 1^{\prime \prime}$ from the second brightest star, \# 14 with $V=16.60$, $B-V=-0.09$, and $V-R=-0.06$ mag. Interestingly, the $V$ and $B-V$ magnitudes for star \#17 agree well with Oey (1996)'s results. We notice that the present higher resolution data reduce the brightness of $\mathrm{Sk}-71^{\circ} 51$ by 0.14 mag with respect to (Isserstedt 1975): $V=12.71, B-V=-0.09$, $U-B=-1.00 \mathrm{mag}$.

\subsection{Spectral types}

The stars for which the spectroscopy was obtained are indicated in Fig. 2, while the spectrograms are displayed in Figs. 9 and 10. The spectral classification was performed without knowledge of the stellar identifications or locations, with reference to the criteria and atlas of Walborn \& Fitzpatrick (1990). The results are summarized in Table 4 which also gives

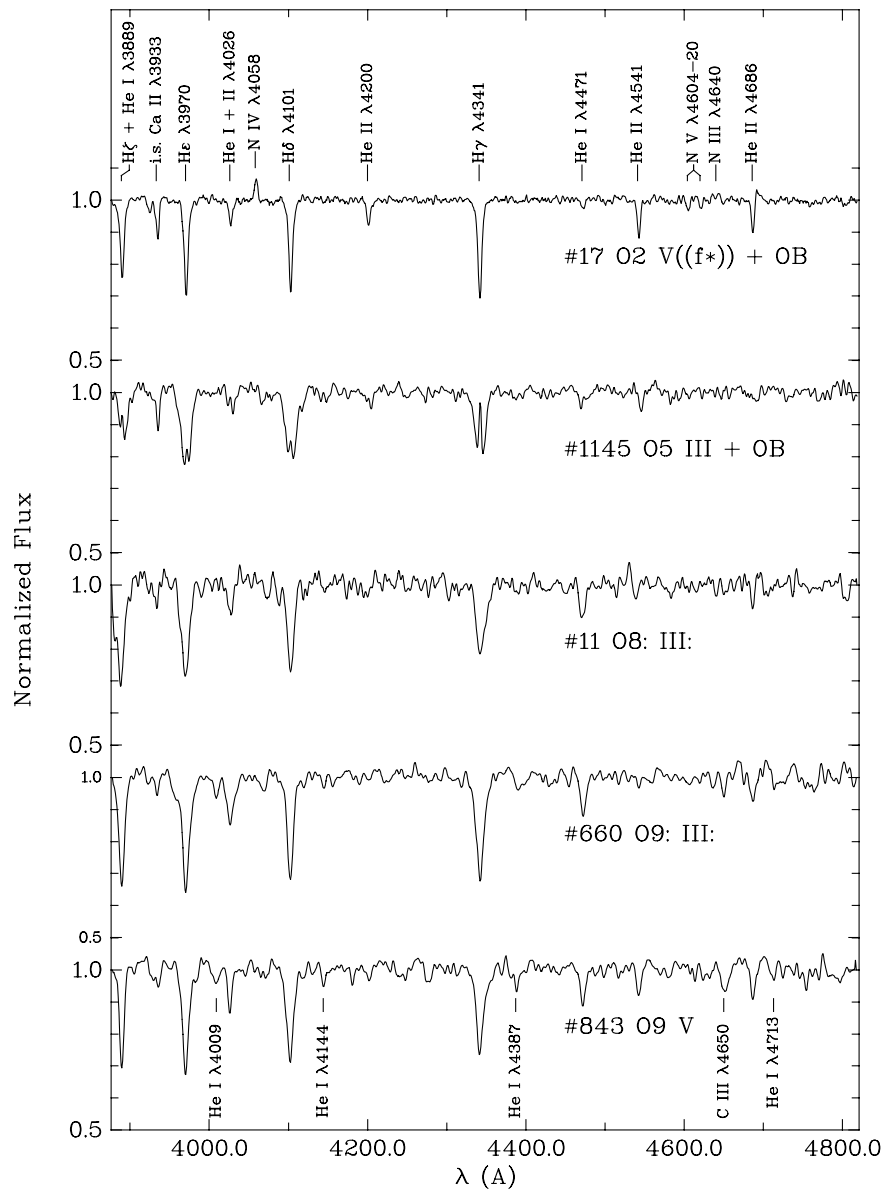

Fig. 9. Spectra of O type stars in the LMC H II region N214C.

the corresponding photometric and astrometric information. In the following some details of the two most interesting cases are given. 


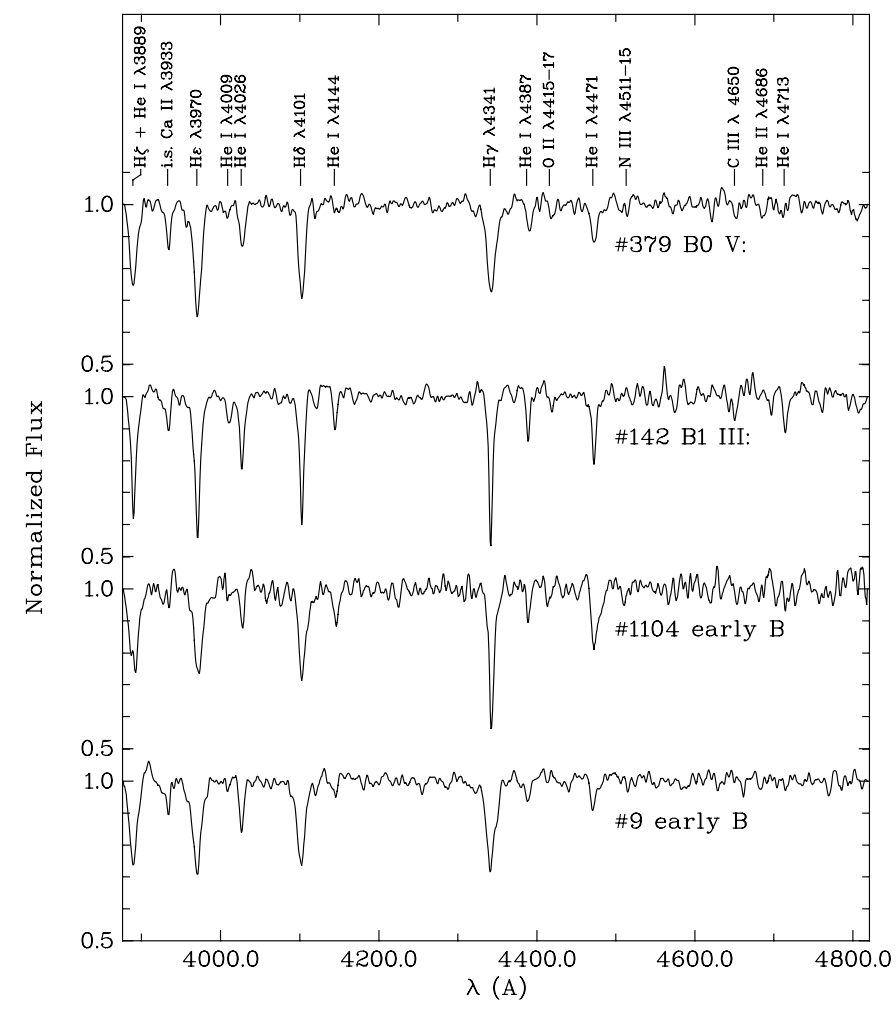

Fig. 10. Spectra of B type stars in the LMC H II region N 214C.

\subsubsection{Star $\mathrm{Sk}-71^{\circ} 51$}

Star \#17 is the brightest component of the $\mathrm{Sk}-71^{\circ} 51$ cluster (Table 3). The spectral classification of $\mathrm{Sk}-71^{\circ} 51$ has changed several times as a function of the quality of the spectra used. Initially it was classified as an early type dwarf, O4 V (Conti et al. 1986), but subsequently was revised to an earlier giant O3 III(f*) (Garmany \& Walborn 1987). More recently, $\mathrm{Sk}-71^{\circ} 51$ was classified as $\mathrm{O} 3 \mathrm{~V}\left(\left(\mathrm{f}^{*}\right)\right)$ by Walborn et al. (2002). The present higher $\mathrm{S} / \mathrm{N}$ ratio spectra indicate an even earlier main sequence star of type $\mathrm{O} 2 \mathrm{~V}\left(\left(\mathrm{f}^{*}\right)\right)$ due to a large $\mathrm{N}$ IV $(\lambda 4058) / \mathrm{N}$ III $(\lambda 4640)$ ratio. Note also the presence of He II $\lambda 4686$ P Cyg profile. The presence of the He I 4471 line suggests an OB companion; it is quite possible that the $\mathrm{He}$ I line arises in the close resolved companions, similarly to the case of LH10-3209 (Walborn et al. 1999). However, contamination by unresolved close components can not be excluded. Assuming an intrinsic color of $E(B-V)=-0.32$ mag for early O type stars (Walborn et al. 2002) and a distance modulus of $18.6 \mathrm{mag}$, the absolute magnitude of star \# 17 is $M_{V}=-6.28$.

\subsubsection{Star \# 1145}

Of spectral type $\mathrm{O} 5 \mathrm{III}+\mathrm{OB}$, this is the brightest star $(V=$ $14.96, B-V=-0.06 \mathrm{mag}$ ) apparently associated with the blob. The doubled line profiles in its spectrogram suggest a spectroscopic binary with $\delta v \sim 530 \mathrm{~km} \mathrm{~s}^{-1}$. The companion dominates at He I $\lambda 4471$ but the primary at He I + II $\lambda 4026$.

\section{Discussion}

\subsection{Stellar content}

The H II region N 214C lies in the OB association LH 110 of size $3^{\prime} \times 2^{\prime}$ (Lucke \& Hodge 1970), in which the latter authors detected seven $\mathrm{OB}$ candidates. The color-magnitude diagram obtained for 2341 stars of the OB association is displayed in Fig. 3. Overplotted are also the isochrones with ages $1 \mathrm{Myr}$, $1 \mathrm{Gyr}$, and $10 \mathrm{Gyr}$ for metallicity $Z=0.008$ obtained by Lejeune \& Schaerer (2001). Two main stellar populations show up in this diagram: a main sequence and an evolved component. The main sequence, well fitted by an isochrones of age 1 Myr with a reddening of $A_{\mathrm{V}}=0.5 \mathrm{mag}$, is made up of stars with initial masses from $\sim 80 M_{\odot}$ to $\sim 4 M_{\odot}$ and possibly as low as $\sim 2 M_{\odot}$. The color spread for the lower mass main sequence stars may be due to either the reddening effect or contamination by the evolved stars.

The evolved population consists of stars which are well fitted by a $1 \mathrm{Gyr}$ isochrone, corresponding to a turnoff mass between 1.9 and $2.0 M_{\odot}$. Stars less massive than the turnoff mass have $(B-V)$ colors between 0.0 and $0.5 \mathrm{mag}$, whereas more massive stars are centered on $(B-V)=\sim 1 \mathrm{mag}$. Note that the 10 Gyr track is tangent to the redder border of the evolved population, probably indicating that the bulk of the stars are better fitted by a much younger, $1 \mathrm{Gyr}$ isochrones.

Star \# 709 is evolved, as indicated by its spectrum presenting features typical of evolved low-mass stars. This result is supported by the position of the star on the color-magnitude diagram. Its absolute magnitude, -2.7 , would point to a latetype $\mathrm{M}$ giant. This star is quite interesting since it lies on the face of the $\mathrm{H}$ II region N $214 \mathrm{C}$ not far from the $\mathrm{Sk}-71^{\circ} 51$ cluster. Generally speaking, several stars seen directly toward N 214C belong to the evolved population. Do they really pertain to this region, or are they field stars? Radial velocity determinations are necessary to answer this question. Another remarkable case is star \# 437, which has the reddest colors of the sample (see also Table 4). It lies toward the loop structure of N214C (Sect. 3.1) where the reddening, while not negligible, is not enough to explain the strong colors measured for \# 437. Comparison with the intrinsic $B-V$ and $V-R$ colors (Houdashelt et al. 2000) suggests an evolved low-mass, field star likely affected by local extinction.

The fact that the H II blob lies toward the northern outskirts of $\mathrm{N} 214 \mathrm{C}$ and the compact $\mathrm{H}$ II regions N214H-1, 2, and 3 are situated in that area, suggests that the detected molecular cloud (Israel et al. 1993; Heikkila et al. 1998) is in contact with the ionized gas in that direction. This assumption agrees with the observation that stars \# 1882 and \# 1846 have a red color (Fig. 3). Note also that stars \# 1936 and more especially \# 1964, the latter situated in the diffuse, and maybe older $\mathrm{H}$ II region $\mathrm{N} 214 \mathrm{H}-4$, have a smaller reddening, because they may be less affected by the molecular cloud.

The upper part of the main sequence is populated by massive $\mathrm{O}$ and $\mathrm{B}$ types several of which have been classified in this work. These stars, seen on the face of the H II region N 214C, are associated with the $\mathrm{H}$ II region and therefore contribute to its ionization. Their initial masses are $<40 M_{\odot}$, with a very 
Table 4. Spectral classication and photometry of interesting stars in LH 110.

\begin{tabular}{ccccrccc}
\hline \hline Star & $\begin{array}{c}\alpha \\
(\mathrm{J} 2000)\end{array}$ & $\begin{array}{c}\delta \\
(\mathrm{J} 2000)\end{array}$ & $\begin{array}{c}V \\
(\mathrm{mag})\end{array}$ & $\begin{array}{r}B-V \\
(\mathrm{mag})\end{array}$ & $\begin{array}{c}V-R \\
(\mathrm{mag})\end{array}$ & Spectral type & Notes \\
\hline 9 & $05: 41: 38.48$ & $-71: 19: 59.1$ & 15.96 & -0.14 & -0.07 & early B & \\
11 & $05: 41: 37.60$ & $-71: 19: 58.6$ & 15.70 & -0.11 & -0.06 & O8: III: & Main component of Sk $-71^{\circ} 51$ \\
17 & $05: 41: 39.62$ & $-71: 19: 56.4$ & 12.85 & -0.15 & -0.06 & O2 V((f*)) + OB & \\
142 & $05: 41: 42.54$ & $-71: 21: 19.4$ & 15.32 & -0.12 & -0.03 & B1 III: & \\
325 & $05: 41: 45.19$ & $-71: 20: 54.2$ & 14.81 & -0.14 & -0.03 & & \\
379 & $05: 41: 48.37$ & $-71: 20: 46.1$ & 15.40 & -0.11 & -0.03 & B0 V: & \\
437 & $05: 41: 26.14$ & $-71: 20: 37.8$ & 16.07 & 2.19 & 1.22 & & \\
660 & $05: 41: 44.53$ & $-71: 20: 06.8$ & 14.92 & -0.15 & -0.03 & O9: III: & late-type \\
709 & $05: 41: 42.29$ & $-71: 19: 59.7$ & 15.85 & 0.83 & 0.55 & O9 V & Associated with the Blob \\
843 & $05: 41: 41.70$ & $-71: 19: 40.6$ & 15.53 & -0.16 & -0.04 & & Seen toward the western lobe \\
1104 & $05: 41: 59.18$ & $-71: 19: 20.4$ & 20.01 & 0.37 & 0.18 & early B & Seen toward the center of the Blob \\
1121 & $05: 41: 36.51$ & $-71: 19: 05.8$ & 16.91 & 0.03 & 0.12 & & Seen toward the eastern lobe \\
1132 & $05: 41: 38.16$ & $-71: 19: 04.3$ & 19.41 & 0.05 & 0.12 & Associated with the Blob \\
1136 & $05: 41: 39.30$ & $-71: 19: 03.9$ & 16.37 & 1.66 & 0.98 & & Associated with N 214H-3 \\
1145 & $05: 41: 37.68$ & $-71: 19: 03.2$ & 14.96 & -0.06 & 0.04 & O5 III + OB & Associated with N 214H-3 \\
1846 & $05: 41: 23.32$ & $-71: 17: 32.8$ & 18.19 & 0.99 & 0.56 & & See Fig. 7 \\
1864 & $05: 41: 22.89$ & $-71: 17: 29.8$ & 15.68 & -0.00 & 0.01 & & Associated with N 214H-2 \\
1882 & $05: 41: 25.43$ & $-71: 17: 27.2$ & 15.03 & 0.71 & 0.41 & & Associated with N 214H-4 \\
1936 & $05: 41: 22.58$ & $-71: 17: 19.2$ & 17.28 & 0.22 & 0.18 & & Associated with N 214H-4 \\
1964 & $05: 41: 26.39$ & $-71: 17: 14.8$ & 16.81 & 0.08 & 0.13 & & \\
2065 & $05: 41: 26.61$ & $-71: 16: 59.5$ & 14.84 & -0.10 & -0.06 & &
\end{tabular}

remarkable exception: star \# 17 with a mass of $\sim 80 M_{\odot}$ if single. This main component of the $\mathrm{Sk}-71^{\circ} 51$ cluster is a very hot star of type $\mathrm{O} 2 \mathrm{~V}\left(\left(\mathrm{f}^{*}\right)\right)$, recently introduced by Walborn et al. (2002). This type is very rare since so far only a dozen members have been identified. Should star \# 17 not be fully resolved, as implied by the presence of He I $\lambda 4471$ in its spectrum, its mass must be smaller than $80 M_{\odot}$. Note however that the later-type OB signature may be due to stars \# 14 or \# 21 lying 1'.2 and 1". 4 respectively from \# 17 and not to a binary companion. In that case, the estimated mass of $\sim 80 M_{\odot}$ based only on photometry, will be maintained. Radial velocity observations may elucidate the situation. Anyhow, star \# 17, as the main excitation source of $\mathrm{N} 214 \mathrm{C}$, should have a powerful wind, as revealed by the presence of wind and shock features created in the nebula around the star (Sect. 3.1).

The blue star \# 325 associated with the south-eastern extension of N 214C must be an $\mathrm{O}$ type of initial mass $30-40 M_{\odot}$, as indicated by the color-mag diagram. Although we do not have spectroscopic observations, the fact that the star is surrounded by [O III] emission in a region globally dominated by $\mathrm{H} \alpha$ emission supports an $\mathrm{O}$ type characteristic. Note that stars \# 379 and \# 142, which are classified B0 V: and B1 III: (Fig. 10), Table 4 and lie toward the same region as \# 325 , are not associated with [O III] emission due to the lack of sufficiently hard ionizing photons in B stars.

\subsection{The peculiar Blob}

The compact H II nebula located $\sim 60^{\prime \prime}$ north of Sk $-71^{\circ} 51$ has a striking spherical shape of $\sim 5^{\prime \prime}(\sim 1.3 \mathrm{pc})$ in radius. Its mass would be $\sim 100 M_{\odot}$, assuming a sphere of $1 \mathrm{pc}$ in radius filled with atomic hydrogen of density $1000 \mathrm{~cm}^{-3}$. The excitation of the blob may be mainly due to star \# 1145, type O5 III + OB, which probably lies outside the sphere. The fact that the bright emission peak, where the $[\mathrm{O} \mathrm{III}] / \mathrm{H} \beta$ ratio has its highest value, lies close to \# 1145 confirms the exciting role of this star. This result is also in line with high-resolution radio continuum observations at $3 \mathrm{~cm}(8.6 \mathrm{GHz})$ and $6 \mathrm{~cm}(4.8 \mathrm{GHz})$ obtained using the Australia Telescope Compact Array (Indebetouw et al. 2004). These observations indicate the presence of a compact, ionized region (B0542-7121) at the position of the blob with radio continuum fluxes $19 \pm 2$ and $18 \pm 1.5 \mathrm{mJy}$ at 3 and $6 \mathrm{~cm}$ respectively. These measurements correspond to a Lyman continuum flux of $4.80 \times 10^{48}$ photons s ${ }^{-1}$ assuming that the $\mathrm{H}$ II region is ionization bounded. The exciting star needed to provide this flux is about O9 V type (Vacca et al. 1996). The discrepancy between this estimate and the spectral classification (O5 III star) can be explained by the fact that the star is outside the blob, so only a fraction of its radiation intercepts in. In case an ionizing source is hidden in the blob the extinction should be so strong that no Lyman continuum photons can escape. The radio continuum observations confirm also that star \# 1104, an early B, does not have a major role in the ionization of the blob. On the other hand, there is a faint star \# 1132 seen toward the center of the blob in the dark opening, but this is most likely a foreground, blue star of $V=19.41, B-V=0.05 \mathrm{mag}$.

The fact that no hidden exciting source is expected does not preclude the presence of an internal infrared object. And, interestingly, a strong IRAS source, 05423-7120, coincides with the blob (Indebetouw et al. 2004). The measured fluxes $\left(F_{12}=\right.$ $0.84 \mathrm{Jy}, F_{25}=3.91 \mathrm{Jy}, F_{60}=45.74 \mathrm{Jy}$, and $\left.F_{100}<110.80 \mathrm{Jy}\right)$ correspond to a luminosity of $\sim 2 \times 10^{5} L_{\odot}$ as derived from the $60 \mu \mathrm{m}$ flux. This estimate implies a massive heat source, equivalent to an embedded $\sim 07 \mathrm{~V}$ star of mass $\sim 40 M_{\odot}$. The IRAS data can be fitted by a blackbody with $T=60 \mathrm{~K}$ whose maximum is centerd on $\sim 60 \mu \mathrm{m}$. This suggests that there is indeed 
an embedded massive star not just a cold collapsing gas clump whose spectral energy distribution would probably peak at or beyond $100 \mu \mathrm{m}$. There is however an excess emission at $12 \mu \mathrm{m}$. Although the excess in some cases could be due to a component of very small grains or polycyclic aromatic hydrocarbons (Degioia-Eastwood 1992; Bell et al. 2002), it could also be due to the presence of a hotter source (Wolf-Chase et al. 2003). The latter suggestion is in line with the expectation that the putative object is most likely not isolated but embedded in an infrared cluster. Alternatively, an accreting protostar cannot be ruled out. In that case a very massive protostar $\left(\sim 100 M_{\odot}\right)$ with a high accretion rate $\left(\sim 10^{-3} M_{\odot} \mathrm{yr}^{-1}\right)$ is required. Another possibility would be a trapped ultracompact H II region created immediately following high mass star formation (Keto 2002). This would imply a low radio luminosity compared to the total luminosity reradiated by dust, suggesting also that the object might be so heavily obscured that it emits mainly in far infrared. If this putative object exists, it would be interesting to determine its near- and mid-infrared properties using high spatial resolution observations.

The compact $\mathrm{H}$ II region discovered in N214C may be a newcomer to the family of HEBs (High Excitation Blobs) in the Magellanic Clouds, the first member of which was detected in LMC N 159 (Heydari-Malayeri et al. 1999b and references therein). In contrast to the typical H II regions of the Magellanic Clouds, which are extended structures spanning several minutes of arc on the sky (more than $50 \mathrm{pc}$ ) and powered by a large number of hot stars, HEBs are dense, small regions usually $5^{\prime \prime}$ to $10^{\prime \prime}$ in diameter ( 1 to $3 \mathrm{pc}$ ). Moreover, they often form adjacent to or apparently inside the typical giant $\mathrm{H}$ II regions, and rarely in isolation. They are generally affected by significant amounts of local dust. The formation mechanisms of these objects are not yet well understood, in the sense that we do not know which particular conditions can give rise to them adjacent to typical Magellanic Cloud H II regions. One thing seems however sure, they represent the youngest massive stars of their OB associations. So far only a half-dozen of them have been detected (SMC N 81, N88A; LMC N 159, N 83B, N 11A, N 160A1 \& A2) and studied using the Hubble Space Telescope (Heydari-Malayeri et al. 1999c,a,b, 2001b,a, 2002a,b). But the exciting stars of the tightest or youngest members of the family remain undetected even with the $H S T$ angular resolution in the visible.

The unusual spherical shape of the blob may be due to lack of angular resolution, if we compare it with the Galactic H II region NGC 2024 (Flame nebula), which has a linear radius of $\sim 1.5 \mathrm{pc}$, comparable to the HII blob. The optical image of NGC 2024 also shows a central elongated obscuration in the north-south direction (see Lenorzer et al. 2004, and references therein). There is a B-type infrared cluster behind the dust lane, and recently Bik et al. (2003) estimated a spectral type of $\sim 08$ for the dominant source of ionizing flux for the H II region. In spite of these apparent similarities, two important differences distinguish the N214C blob from NGC 2024. The most massive star of the latter is an O8 type accompanied by a cluster of $\mathrm{B}$ types, while the blob is associated with a more massive star of type O5 III + OB (\# 1145). Moreover, the blob lies in a prominent region which has formed several $\mathrm{O}$ types and more especially a very rare, massive, and hot star of type $\mathrm{O} 2 \mathrm{~V}$. These characteristics call for high-resolution infrared observations of the blob in order to investigate deep into the blob, specifically behind the absorption lane. It would be very interesting if the embedded stellar population of the blob turns out to be similar to that of NGC 2024. This will imply that sharply different environmental factors (for instance metallicity and initial mass function) can bring about similar stellar populations under certain conditions. Knowing these particular conditions will be important for better understanding star and cluster formation.

The ridge feature upon which the H II blob seems to lie, may in fact be an ionization front moving northward into the molecular cloud. If this assumption is right, the blob may have resulted from massive star formation following the collapse of a thin shell of neutral matter accumulated between the shock and ionization fronts, as predicted by the sequential star formation scenario (Elmegreen \& Lada 1977). A list of carefully selected Galactic candidate regions which are likely to be examples of this star formation process is presented by Deharveng et al. (2005). Based on HST observations, this scenario has also been suggested for LMC N 83B (Heydari-Malayeri et al. 2001b). Since star \# 1145 and the blob lie apparently close to each other, one can raise the question whether that star also is triggered by the ionization front of star \# 17. Star \# 1145 (O5 III + $\mathrm{OB})$ is less massive than \# $17(\mathrm{O} 2 \mathrm{~V}+\mathrm{OB})$ and should naturally evolve more slowly than the latter. Yet the luminosity class of \# 1145 seems to imply the contrary. It should be underlined that the luminosity class III means that the absorption feature He I $\lambda 4686$ is filled in by emission. But this description of the spectrum could have different physical causes. Usually that is due to higher luminosity, but anything that produces the He I $\lambda 4686$ emission will fill it in, e.g. loose material/colliding winds in the binary. Therefore, one cannot rule out the possibility that \# 1145 be a second generation star triggered by \# 17 .

Figure 5 reveals another ridge southeast of N214C, suggesting that the $\mathrm{H}$ II region is in contact with a molecular cloud also in that direction. Since the massive stars \# 142, \# 325, and \#379 are apparently situated beyond the south-eastern ridge, the question of sequential star formation can similarly be raised for them.

Acknowledgements. We are grateful to Drs. P. Magain, F. Courbin and their team for giving us access to their new deconvolution code and for their hospitality at their Institute in Liège. We are also indebted to Dr. J. Walsh (ST-ECF) for his tool, specres, and the useful advices about its use. We are also thankful to Dr. L. Germany (ESO, NTT) for providing valuable customized calibration data, which greatly improved our data reduction. We are also grateful to Drs. Lise Deharveng (Laboratoire d'Astrophysique de Marseille), Thibaut Le Bertre (LERMA, Paris Observatory), and Hans Zinnecker (Astrophysikalisches Institut Potsdam) for discussions. We would like also to thank the referee, Dr. Paul Crowther, for helpful remarks and comments.

\section{References}

Bell, E. F., Gordon, K. D., Kennicutt, R. C., \& Zaritsky, D. 2002, ApJ, 565, 994

Bertin, E., \& Arnouts, S. 1996, A\&AS, 117, 393 
Bik, A., Lenorzer, A., Kaper, L., et al. 2003, A\&A, 404, 249

Caplan, J., \& Deharveng, L. 1985, A\&AS, 62, 63

Caplan, J., \& Deharveng, L. 1986, A\&A, 155, 297

Chin, Y.-N., Henkel, C., Whiteoak, J. B., et al. 1997, A\&A, 317, 548

Conti, P. S., Garmany, C. D., \& Massey, P. 1986, AJ, 92, 48

Davies, R., Eliott, K., \& Meaburn, J. 1976, Mem. R. Astron. Soc., 81, 89

Degioia-Eastwood, K. 1992, ApJ, 397, 542

Deharveng, L., Zavagno, A., \& Caplan, J. 2005, A\&A, 433, 565

Elmegreen, B. G., \& Lada, C. J. 1977, ApJ, 214, 725

Garmany, C. D., \& Walborn, N. R. 1987, PASP, 99, 240

Groenewegen, M. A. T., \& Oudmaijer, R. D. 2000, A\&A, 356, 849

Heikkila, A., Johansson, L. E. B., \& Olofsson, H. 1998, A\&A, 332, 493

Henize, K. G. 1956, ApJS, 2, 315

Heydari-Malayeri, M., Charmandaris, V., Deharveng, L., Rosa, M. R., \& Zinnecker, H. 1999a, A\&A, 347, 841

Heydari-Malayeri, M., Rosa, M. R., Charmandaris, V., Deharveng, L., \& Zinnecker, H. 1999b, A\&A, 352, 665

Heydari-Malayeri, M., Rosa, M. R., Zinnecker, H., Deharveng, L., \& Charmandaris, V. 1999c, A\&A, 344, 848

Heydari-Malayeri, M., Charmandaris, V., Deharveng, L., et al. 2001a, A\&A, 372, 527

Heydari-Malayeri, M., Charmandaris, V., Deharveng, L., et al. 2001b, A\&A, 372, 495

Heydari-Malayeri, M., Charmandaris, V., Deharveng, L., et al. 2002a, A\&A, 381, 941
Heydari-Malayeri, M., Rosa, M. R., Schaerer, D., Martins, F., \& Charmandaris, V. 2002b, A\&A, 381, 951

Heydari-Malayeri, M., Meynadier, F., \& Walborn, N. R. 2003, A\&A, 400, 923

Houdashelt, M. L., Bell, R. A., Sweigart, A. V., \& Wing, R. F. 2000, AJ, 119, 1424

Indebetouw, R., Johnson, K. E., \& Conti, P. 2004, AJ, 128, 2206

Israel, F. P. 1997, A\&A, 328, 471

Israel, F. P., Johansson, L. E. B., Lequeux, J., et al. 1993, A\&A, 276, 25

Isserstedt, J. 1975, A\&AS, 19, 259

Keto, E. 2002, ApJ, 580, 980

Lejeune, T., \& Schaerer, D. 2001, A\&A, 366, 538

Lenorzer, A., Bik, A., de Koter, A., et al. 2004, A\&A, 414, 245

Lucke, P. B., \& Hodge, P. W. 1970, AJ, 75, 171

Lucy, L. B., \& Walsh, J. R. 2003, AJ, 125, 2266

Magain, P., Courbin, F., \& Sohy, S. 1998, ApJ, 494, 472

Magain, P., Courbin, F., Sohy, S., Gillon, M., \& Letawe, G. 2005, in preparation

Oey, M. S. 1996, ApJS, 104, 71

Vacca, W. D., Garmany, C. D., \& Shull, J. M. 1996, ApJ, 460, 914

Walborn, N. R., \& Fitzpatrick, E. L. 1990, PASP, 102, 379

Walborn, N. R., Drissen, L., Parker, J. W., et al. 1999, AJ, 118, 1684

Walborn, N. R., Howarth, I. D., Lennon, D. J., et al. 2002, AJ, 123, 2754

Wolf-Chase, G., Moriarty-Schieven, G., Fich, M., \& Barsony, M. 2003, MNRAS, 344, 809 